\title{
Getting Real: The Maryland Healthcare Ethics Committee Network's COVID-19 Working Group Debriefs Lessons Learned
}

\author{
Norton Elson ${ }^{1} \cdot$ Howard Gwon ${ }^{2}$ (D) Diane E. Hoffmann ${ }^{3}$ (1) . \\ Adam M. Kelmenson ${ }^{4}\left({ }^{\circ}\right.$. Ahmed Khan ${ }^{5}$. Joanne F. Kraus ${ }^{6} \oplus$. \\ Casmir C. Onyegwara ${ }^{7} \cdot$ Gail Povar $^{8} \cdot$ Fatima Sheikh $^{9} \cdot$ Anita J. Tarzian $^{10}(1)$
}

Accepted: 11 January 2021 / Published online: 13 February 2021

(C) This is a U.S. government work and not under copyright protection in the U.S.; foreign copyright protection may apply 2021

\begin{abstract}
Responding to a major pandemic and planning for allocation of scarce resources (ASR) under crisis standards of care requires coordination and cooperation across federal, state and local governments in tandem with the larger societal infrastructure. Maryland remains one of the few states with no state-endorsed ASR plan, despite having a plan published in 2017 that was informed by public forums across the state. In this article, we review strengths and weaknesses of Maryland's response to COVID-19 and the role of the Maryland Healthcare Ethics Committee Network (MHECN) in bridging gaps in the state's response to prepare health care facilities for potential implementation of ASR plans. Identified "lessons learned" include: Deliberative Democracy Provided a Strong Foundation for Maryland's ASR Framework; Community Consensus is Informative, Not Normative; Hearing Community Voices Has Inherent Value; Lack of Transparency \& Political Leadership Gaps Generate a Fragmented Response; Pandemic Politics Requires Diplomacy \& Persistence; Strong Leadership is Needed to Avoid Implementing ASR ... And to Plan for ASR; An Effective Pandemic Response Requires Coordination and InformationSharing Beyond the Acute Care Hospital; and The Ability to Correct Course is Crucial: Reconsidering No-visitor Policies.
\end{abstract}

Keywords COVID-19 $\cdot$ Pandemic $\cdot$ Triage $\cdot$ Resource allocation $\cdot$ Ethics committee $\cdot$ Community

Anita J. Tarzian

atarzian@law.umaryland.edu

Extended author information available on the last page of the article 


\section{Introduction}

Until 2020, the United States had avoided widespread ravages of a modern-day pandemic. While the Federal and State governments had done some planning, including tabletop exercises, testing the assumptions embedded in the hypothetical scenarios has been a real-life challenge. As COVID-19 case numbers began to rise, healthcare providers across Maryland wondered how a hypothetically derived and theoretically supported allocation of scarce resources (ASR) plan would hold up in practice. Responding to a major pandemic requires coordination and cooperation across sectors: federal, state and local governments in tandem with larger societal infrastructure. State governments are tasked with playing a major role in pandemic response-redundancy, inefficiency, and inequity may result without centralized state oversight and broad transparency. Here, we review strengths and weaknesses of Maryland's response to COVID-19 and the role of the Maryland Healthcare Ethics Committee Network (MHECN) in bridging gaps in the state's response to prepare health care facilities for potential implementation of ASR plans.

\section{Lesson \# 1: Deliberative Democracy Provided a Strong Foundation for Maryland's ASR Framework}

A Johns Hopkins -led project team comprising Johns Hopkins Medicine, the Berman Institute for Bioethics, and the University of Pittsburgh Medical Center held Deliberative Democracy forums across Maryland between 2012 and 2014 to consider the role of the community in determining which ethical principles should guide decision-making about scarce life-saving resource allocation in a catastrophic health emergency (Biddison-Daugherty et al. 2017). This work was funded by a grant from the federal Office of the Assistant Secretary for Preparedness and Response. The project team held eight forums with lay people and seven forums with disaster and healthcare workers. Primary data sources were pre- and post-surveys, notes from group deliberations, and exit interviews with selected participants. A total of 324 individuals participated, representing a diverse selection by race, education level, socioeconomic status, and religious affiliation. Forum participants were given materials to review in advance that discussed hypothetical emergency pandemic scenarios with insufficient ventilators across Maryland hospitals. In the half-day moderated forums, participants weighed which ethical principle(s) out of six should determine the patients that receive priority ventilator access: (1) those most likely to survive the current illness; (2) those who could go on to live the most life years; (3) those who could go on to live through more life stages; (4) those who would be of most value to others (e.g., first responders, parents/caregivers, essential workers); (5) those who arrived earliest to the facility (i.e., first-come, first-served), or (6) equal priority for all patients (e.g., lottery).

Anticipatory public discussion about ASR in a crisis is critical because: (1) the public will bear the consequences of these decisions; (2) the public's values are 
central to choosing among multiple ethically permissible ASR approaches; (3) a successful allocation process requires public trust and cooperation, both of which are more likely if the process has been transparent, inclusive, and impartial; (4) knowledge of public perspectives and morality better prepares health authorities to develop preparedness plans, allocation guidance and risk communication for contentious issues; and (5) advanced discussion and planning are essential because there is little time for in-depth deliberations with the public, government, legal community and hospitals in the midst of a pandemic (Daugherty Biddison et al. 2014, p. 778).

The Deliberative Democracy approach (as opposed to polling or inviting the public to comment on drafted policy) provides materials for participants to review, time for deliberation, a structure to moderate discussion, and multi-disciplinary experts to present scenarios and basic medical facts. This format ensures participants reach the same starting point, fostering open and informed conversations from multiple perspectives based on the best information available. The forum approach allows participants to express their values and reasoning behind principle selection, while also providing opportunities for participants to change their views after listening to other members of the community. For example, many participants were initially concerned about or opposed to removing a ventilator from one patient in favor of another in the context of pandemic triage, considering this morally unacceptable. However, about half of the respondents changed their mind on this issue by the end of the half-day forum discussion, recognizing that such an approach would likely save more lives (although many remained concerned about bias influencing these decisions) (Daugherty Biddison et al. 2014). While the labor-intensive nature of this approach precluded representing every sector of the community, it has been recognized as an exemplar of community engagement surrounding ASR in crisis standards of care (CSC).

\section{Lesson \#2: Community Consensus is Informative, Not Normative}

While the goal of the Deliberative Democracy project was to inform the ethical principles supporting Maryland's ASR framework used during CSC, incorporating descriptive data into normative guidelines proved challenging. For example, based on participants' appraisals of the ethical principle to use first when allocating ventilators during periods of scarcity in a pandemic, the three ethical principles most commonly ranked first were: saving the most life-years $(37.6 \%)$, surviving the current illness $(31.2 \%)$, and value to others $(17.2 \%)$. The same three principles, ranked slightly differently, bested the others (proportion of respondents who selected "often" or "always") when participants were asked how often each of the six principles should be used (surviving the current illness: $71.1 \%$; live longer: $56.3 \%$; value to others: 48.1\%) (Daugherty Biddison et al. 2014, 2018). Reconciling these results with other ethical analyses involving ASR processes proved difficult. For example, the bioethics literature in general advocates that while frontline workers should be protected, it should not be in the form of an ASR triage score that gives them priority access to scarce resources (Sveen and Antommaria 2020). Maryland's ASR 
framework ultimately excluded the principle of value to others, despite forum results placing this principle among the top three principles selected. However, the resulting ASR framework did preserve a point credit for women gestating a fetus with a detectable heartbeat as recognition that a mother is of instrumental value to her newborn (Biddison-Daugherty 2017).

Similar to other ASR frameworks (Antommaria et al. 2020), Maryland's framework adopted Sequential Organ Failure Assessment (SOFA), a scoring system developed to predict mortality over the short-term for ICU patients. SOFA can help assess organ dysfunction or failure, with a higher score indicating poor physiologic function in multiple organs, which is associated with a higher predicted mortality. Worries remain over whether SOFA is the best predictor of benefit from a ventilator as well as over the lack of data for short-term survival from COVID-19. Furthermore, there are concerns about computation errors that may disadvantage persons with certain chronic conditions. For example, persons with asymptomatic chronically elevated bilirubin (Gilbert's syndrome) may receive a poorer liver component score than their actual liver function warrants; those with disrupted renal dialysis access may have higher creatinine levels that could yield poorer overall SOFA scores, and those with a history of a chronic neurologic condition such as Cerebral Palsy or paralysis may receive a poorer score for central nervous system function without accommodating for their stable baseline condition. However, no other scientifically rigorous scoring system has been developed that is feasible to implement in the context of pandemic triage that would correct for certain prior health care inequities.

Maryland's ASR framework accounted for community members' concerns about disadvantaging those who, as a result of systemic racism and inequities in receipt of health care, showed shorter life expectancy and would thus be de-prioritized if subjected to a scoring method that quantified comorbidities. This was accomplished by assigning a lower-priority score only to individuals with less than a one-year life expectancy due to certain underlying conditions, and no triage score adjustment based on quantifying comorbidities. Thus, the community value of "surviving the current illness" (expanded to a one-year interval) was prioritized over the community value of "life years saved." Three other ethical principles were incorporated into Maryland's 2017 ASR framework as tie-breakers: life stages (allocating points by age, including prioritizing individuals 0-49 years of age and deprioritizing those 85 years and older); and fair chance via lottery or first-come, first-served methods.

\section{Lesson \#3: Hearing Community Voices Has Inherent Value}

The perception of transparency, inclusivity, and representativeness are arguably the greatest benefits from the public engagement that informed Maryland's 2017 ASR framework (the "2017 Plan"). The ethical principle deemed most important by members of the community—saving the most lives—is prioritized in the 2017 Plan's triage scoring. However, not all community values were retained, and some that were retained have since been criticized (e.g., civil rights advocates challenged the inclusion of age as a tie-breaker and other aspects of the triage score calculation as vulnerable to implicit 
biases that may disadvantage individuals with disabilities). These objections highlight the challenge of using public engagement to inform policy. Not all stakeholders will be represented. Furthermore, stakeholder groups represented by organizations advocating on their behalf may not all share the same position. Consensus arising from discussions among individual citizens may look very different from recommendations arising in response to legal and political advocacy. Broader consensus from triage experts and the bioethics literature must also be considered.

Oberlander and colleagues (2001) described similar challenges related to Oregon's experience using public engagement to guide Medicaid services rationing. While some might view this as a reason why community engagement is not worth the effort, we disagree. Cultivating community voices builds public trust and support, which are essential in pandemic planning and ASR implementation. As Oberlander and colleagues summarized (2001, p. 1586): "The real innovation in Oregon has been drawing on public participation to build public support ...." It was hoped that the same would be said for Maryland's 2017 Plan.

\section{Lesson \#4: Lack of Transparency and Political Leadership Gaps Generate a Fragmented Response}

Unfortunately, there were delays in funding the final phases of the Hopkins ASR project and the ASR framework was not completed until August, 2017. For reasons we are not privy to, no state action was taken to implement the framework's recommendations. Lack of political will foreshadowed problems that thwarted a streamlined state response to the COVID-19 pandemic. Most notable among the problems was the absence of a coherent, consistent and accessible state ASR plan (or more broadly, a CSC plan). This deficiency is apparent in Manchanda et al. (2020) review of state CSC plans in which Maryland is noted as having "none identified."

In a pandemic, difficult decisions are made by clinicians and emergency response workers in real time. Ideally, the state government provides the structure and the assurances necessary for public discussions about these difficult choices. Without statewide dissemination and discussion of an ASR plan, various stakeholder groups (e.g., the disability community, racial/ethnic minorities, the elderly), and health care institutions had inconsistent access to a potential state plan drafted by a group of hospitals in the state (see below). Although public-private partnerships emerged to fill some gaps (e.g., The Baltimore Convention Center Field Hospital operated jointly by the University of Maryland Medical System and Johns Hopkins Hospital, and arrangements with local hotels to provide lodging for individuals testing positive for SARS-CoV-2), a lack of action at the state level created inefficiencies and patchwork policies.

\section{Five Hospital Systems Respond}

In the absence of a state-endorsed ASR plan, shortly before the anticipated April 2020 COVID-19 case surge in Maryland, a group composed of representatives of four major health systems (Johns Hopkins, Lifebridge, MedStar, and the 
University of Maryland) formed in March 2020 to develop an operational ASR plan from Hopkins' 2017 Plan. A fifth hospital system, Luminis, was later added. The " $5 \mathrm{H}$ " group initially focused on allocation strategies for ventilators. A focus on ventilators came in response to data from Italy and elsewhere pointing to estimates that the demand for ventilators would be much higher than their supply across the state (Broadwater 2020; Broadwater and Wood 2020). Consistent with the 2017 Plan, the ethical framework that guided the 5H Plan included the duty to provide care and to steward resources. The $5 \mathrm{H}$ group built on the ethical justifications underlying the 2017 Plan: maximizing benefit by saving as many lives as possible, grounded in substantive and procedural justice.

The 5H group (encompassing about half of the state's hospitals) shared various drafts of their plan with the Governor's office. Their primary goal was to provide an ASR plan for their five hospital systems. A secondary goal was to have their plan adopted by all Maryland hospitals to create consistency across the state in how hospitals addressed ASR during the pandemic. State-wide consistency in ASR implementation could have been accomplished by gubernatorial action via an executive order under Maryland's Catastrophic Health Emergencies Act requiring all hospitals to implement the 5H's ASR Plan, if the need arose to ration critical care resources. The Governor's executive order would have also triggered immunity from civil or criminal liability for any physician who complied with the state-authorized plan. This would include removing a patient with a poor chance of recovery from a ventilator after a specified trial period, without consent, in order to provide it to another patient with a greater likelihood of survival. However, the Governor did not endorse any version of the $5 \mathrm{H}$ Plan or make it available for hospitals to review and plan accordingly. Instead, the Governor decided to put the plan on hold until the situation called for its activation. Unfortunately, this wait-and-see strategy stymied an opportunity to address operational and administrative challenges within and across hospitals and health sector coordination.

\section{Maryland Hospital Association Responds}

The Maryland Hospital Association (MHA), serving its members, distributed a draft of the 5H Plan in May to hospital CEOs after receiving a presentation from members of the 5H group. While MHA's dissemination of an earlier draft of the $5 \mathrm{H}$ Plan allowed some hospitals to plan internally for its execution, coordination both locally and regionally to achieve a standard implementation process was thwarted by lack of central organization at the state level. Unfortunately, several hospitals did not attend the MHA presentation or receive the 5H Plan. One example is a small hospital on Maryland's Eastern Shore. The director of chaplaincy there undertook extensive efforts to locate information about how her hospital should respond if it depleted its available resources to treat patients with COVID19. She was grateful to discover an invitation to join the statewide COVID-19 
working group sponsored by the Maryland Healthcare Ethics Committee Network (MHECN).

\section{State Ethics Network Responds}

MHECN is a membership-supported organization affiliated with the University of Maryland Carey School of Law that has served members of healthcare ethics committees in the state since 1993. It sponsors ethics education programs, distributes a newsletter, promotes networking in healthcare ethics, weighs in on ethical questions and concerns raised by its members, and engages in research and policy initiatives relevant to local clinical practice and healthcare ethics (Tarzian et al. 2006).

About the time that the $5 \mathrm{H}$ group was assembling to enact its plan, MHECN invited interested members to join a COVID-19 working group (WG) to address members' questions about the anticipated COVID-19 case surge. With representatives from both large hospital systems and smaller institutions, as well as outpatient physicians, bioethicists, lawyers, and nursing home and hospice leadership, MHECN provided a forum to raise concerns and problem solve as the challenges of the pandemic became apparent and evolved over time. MHECN's forum proved especially important given government silence in response to the potential need for ASR.

The WG began meeting by ZOOM twice weekly in March, with initial discussions devoted to operationalizing the 2017 Plan with its focus on allocating ventilators if rationing became necessary. The WG learned of the 5H group's work and was ultimately provided a draft of the $5 \mathrm{H}$ plan with a request by the $5 \mathrm{H}$ group not to share it to avoid different versions of the plan "floating around" the state, potentially leading to hospitals applying different criteria. The goal of both the WG and the $5 \mathrm{H}$ group was to have consistent ASR standards and procedures across all hospitals in the state. However, as a group representing five private hospital systems, the $5 \mathrm{H}$ group had no responsibility to share its work with others - again pointing out the challenges of working on a plan divorced from state oversight.

The WG also served as a kind of "safety valve" for staff to share concerns outside of their home institution, whose policies were sometimes opaque to them. For instance, how PPE was allocated at members' home institutions, and on what basis, was unclear to some. WG members also expressed discomfort about teams of medical students and residents who were patently unable to manage safe distancing and consistent masking, clustered as they are in the "hard geography" of the hospital team rooms, where charting, eating, and learning all take place at once. The WG offered its perspective on ways such practices might be modified. A conversation up and down the levels of responsibility is necessary for even something as "simple" as infection control to be implemented meaningfully. Neglecting this is a recipe for deteriorating staff morale, and is disrespectful to staff who deserve the full measure of harm reduction approaches to safeguard their well-being. In situations of stress, asking staff to "just trust us" may only add to their sense of uncertainty, which greatly amplifies anxiety and stress and reduces efficiency that is central to an 
effective pandemic response. WG members reminded those sharing these concerns of best practices for protecting their well-being.

Another example of how the WG was able to fill in informational gaps was lack of awareness of the Maryland Institute for Emergency Medical Services Systems' (MIEMSS) newly-developed online capture of available ICU beds, ventilators, and dialysis units available at hospitals across the state. This database was to be updated several times a day during the COVID surge. Access to this information was central to the question of when a hospital would switch from contingency standards of care to CSC (thus invoking ASR). While individual hospitals' incident command center staff were aware of its existence and how to access it (only particular individuals in the hospital have requisite permissions), this information was not always shared with other frontline staff. Sharing this critical information assuaged concerns among hospital-based WG members that there would be a clear signal when their facility would transition to CSC.

\section{Civil Rights Advocacy Groups Respond}

Leading up to and during the COVID case surge in early 2020, many states reviewed previous ASR plans as a basis to address any possible shortages resulting from the COVID-19 pandemic. Likewise, disability rights advocates reviewed the plans' language to forgo discrimination. Alabama's 2010 "Crisis Standards of Care" plan, for example, specifically precluded access to mechanical ventilators for patients with "profound mental retardation," "moderate to severe dementia," and "severe traumatic brain injury" (Mello et al. 2020, p. e26(2)). In April 2020, the Department of Health and Human Services' Office of Civil Rights (OCR) announced an early resolution of its complaint against Alabama after the state removed rationing guidelines "that allegedly discriminated on the basis of disability and age" (DHHS, April 8,2020 ). Further, OCR voiced concerns that the 2010 Criteria, if used to guide ASR for patients with COVID-19 should that need arise, could "impose blunt age categorizations, such that older persons might automatically be deemed ineligible for lifesaving care without any individualized assessment or examination and based solely on missing a strict age cutoff' (DHHS, April 8, 2020).

To avoid this type of discrimination, the WG requested a meeting with disability rights advocates, who gave feedback on Maryland's 2017 Plan. However, because the $5 \mathrm{H}$ ASR plan was not disseminated by the state and lacked an authoritative "home," no identified entity could centrally receive comments/suggestions. Instead, the WG initiated a meeting at which $5 \mathrm{H}$ representatives heard directly from disability and aging advocates. This meeting fostered interaction with stakeholders and further refinement of the proposed ASR plan that would ordinarily be the province of the state policy process. Embargos on sharing earlier drafts of the $5 \mathrm{H}$ Plan (see Ehmann et al., in press, for a recently published version) and lack of procedures for receiving public feedback revealed the shortcomings of this ad hoc approach. 


\section{Lesson \#5: Pandemic Politics Requires Diplomacy and Persistence}

The MHECN WG realized it would be advantageous if the state took a more active role in making the 5H Plan available to the hospitals and to the public. As a result, the WG leadership met (virtually) with the authors of the 5H Plan and suggested that both groups collaborate. Members of the $5 \mathrm{H}$ Plan were in agreement with the need to make the plan publicly available, but worried about their ability, moving forward, to revise the plan in response to changing data and new information about the virus and its treatment. They considered the plan a living document needing to change as the circumstances warranted.

Both groups (5H and the WG) acknowledged the need to balance "nimbleness and transparency" and thus proposed a path forward that would not require that the Governor, at least initially, adopt the plan. It would simply require that the $5 \mathrm{H}$ Plan be posted on the state department of health's website. Comments by interested persons or organizations could then be forwarded to the $5 \mathrm{H}$ group for their consideration. However, the WG also recommended that if the Governor adopted the $5 \mathrm{H}$ Plan as a state document, the state use the Governor's website and social media to announce the request and provide the details of the specific triage plan in question (e.g., ventilator, dialysis, etc.), and invite comments to the Governor's office. The comment period would be whatever was feasible under the circumstances.

The Governor would also be asked to appoint a statewide "Central Triage Committee" as recommended in the 2017 Plan. In the event of the need to triage, this Committee would "review triage decision-making on a state-wide level," conduct research based on state-wide data collection, and, if necessary, modify allocation algorithms (Daugherty-Biddison et al. 2017, p. 19). Subsequently, the WG drafted a letter to the Governor consistent with this agreement and had it approved by the $5 \mathrm{H}$ group. On August 19, 2020, the letter was delivered to the Governor's office, but as of the writing of this article, no response has been received.

\section{Lesson \#6: Strong Leadership is Needed to Avoid Implementing ASR ... And to Plan for ASR}

The first wave of critically ill COVID-19 patients created an "all hands-on deck" demand for staffed ICU beds to meet the need for "space, staff, and stuff" (Daugherty-Biddison 2017, p. 27). Maryland's Governor managed the pandemic through the expansion of medical capacity and associated supplies and staff in order to avoid having to implement an ASR plan unless absolutely necessary. The action plan was to flatten and lower the transmission curve by containing the spread of the virus through widespread testing, contact tracing, and quarantine procedures to reduce hospitalizations, alongside messaging to encourage the public to don masks and routinely use protective measures (i.e., frequent hand washing, physical distancing, and avoiding crowds) to limit their exposure to the virus.

The plan was to add 6700 beds in 40 days throughout the state. With the help of FEMA funds and reallocation of state monies, additional in-hospital units as well 
as field hospitals, such as the one at the Baltimore Convention center, were created. Additional ventilators from the Federal Government and medical and operational staff from the Army Corps of Engineers augmented the state's resources. More importantly, these efforts allowed the responder community to avoid implementing a rationing plan. Maryland's Governor also purchased test kits from South Korea to boost testing capacity (despite a setback of flawed kits requiring replacement), along with what tests were available in the U.S. This increased testing plan and public compliance helped decrease the state's peak positivity rate from $26.9 \%$ to $3.39 \%$ over 18 weeks (May 1 - September 1). June 17 marked the first time the positivity rate dropped below the $10 \%$ target in every jurisdiction.

Based on the media reports of the state's strategy, the Governor enacted 38 executive orders to reduce the transmission of the virus and to facilitate more efficient treatment of infected individuals (e.g., by allowing health care providers not licensed in Maryland to work in the state and allowing retired health care providers to provide services without reinstating their inactive license). These actions mirror what occurred in New York, New Jersey, and other states in the northeast and upper mid-Atlantic to contain the spread of the virus. However, concerns remained about limited testing capacity and long turnaround periods for results as fall and winter approached, especially absent a coordinated federal response. Furthermore, preparing for the worst (i.e., planning for ASR implementation) was not considered a priority (Sisak 2020).

We believe that in the face of a pandemic of unknown severity, planning for the worst is essential. Keeping the ASR framework out of public view was perhaps intended to minimize panic and unnecessary worry. This approach, however, created other problems. Not having these processes in place before Maryland's April 2020 COVID-19 spikes caused confusion and stymied the coordinated and efficient response that typifies effective disaster planning. This is not a guarantee going forward. Even if viral contagion remains in check, there is value in planning for a "worst case" scenario. "Piloting" ASR practices across facilities and implementing a statewide ASR oversight mechanism could prove useful for future health emergencies in the state. Moreover, although ASR for the Spring 2020 COVID-19 case surge was avoided, the anticipation that it might have been necessary created anxiety among frontline workers who were uncertain how their facility would respond. The ability to reduce uncertainty by "planning for the worst" and thus relieve anxiety among responders during the high-stress milieu of a pandemic is inherently worthwhile.

\section{Lesson \#7: An Effective Pandemic Response Requires Coordination and Information-Sharing Beyond the Acute Care Hospital}

ASR plans have been, and largely remain, hospital centric. It is understandable that hospital systems are initially at the forefront when facing acute shortages of resources to treat critically ill patients. However, it soon became evident to the MHECN WG that other resource shortages affected the COVID-19 pandemic response. The need to expand beyond the focus on ventilator shortages further up 
the chain to directly reduce the need for ventilator allocation quickly became apparent. WG meetings focused on strategies to disseminate information about the state's COVID-19 response in three general areas: (1) minimizing hospital admissions through community initiatives and coordination between acute care facilities (e.g., hospitals) and chronic care facilities (e.g., nursing homes and hospices), (2) clarifying processes for standard, contingency, and crisis standards of care for hospitalized patients, and (3) preventing hospital discharge delays by streamlining processes for post-hospital care (e.g., caring for patients with COVID-19 post-discharge at home, rehabilitation facilities, hospice, and long-term care (LTC) settings) (see Table 1). The diverse representation among WG members meant there was valuable pooled expertise and frontline experience. WG members shared resources not known to all, and MHECN was able to disseminate this information to its members.

\section{Responding to Needs Outside Acute Care Hospital Settings}

Healthcare systems scrambled to offer telehealth, home care, and hospice services, which were critically important in meeting the needs of the community. The state government allied with the private sector to provide housing for homeless individuals. A state 2-1-1 number was advertised to identify individuals at risk and match needs with available resources. The state medical reserve corps and other volunteer services were mobilized (although onboarding/training and matching needs and services could be improved). One success story that spread nationally centered on the Maryland Baptist Aged Home in Baltimore, where no cases of COVID-19 were detected after its director took early precautions to prevent the spread of the virus among its predominantly African American residents (Rodericks June 18, 2020). This provides "proof of concept" that education and early response precludes relying on hospitals and ASR to save lives.

Attention to state-of-the-art advanced care planning and documentation of preferences [via advance directives and accurate Maryland Orders for Life Sustaining Treatment (MOLST) orders] may have reduced the frequency of "standard of care" defaults to aggressive ICU-level care at the end of life when it was inconsistent with an individual's goals of care. In the context of COVID-19, individuals who prefer to remain in the community and in their residential facilities should have that option. Failure to provide this simple matching of needs and resources should be one lesson we carry with us post-pandemic. It will require attention to the conversations patients have with their health care agents regarding goals of care and end-of-life preferences.

The LTC setting is one sector that deserves special consideration. Attention on nursing homes during the COVID-19 pandemic has frequently focused on unacceptably high death rates. This could reflect substandard care or the reality that elderly persons are simply more likely to die from COVID-19, despite the level of medical attention they receive. The death rate for LTC residents who tested positive for SARS-CoV-2 in the U.S. is 25.2\% (Centers for Medicare \& Medicaid Services); the LTC death rate in Maryland is $14.2 \%$ (Maryland Department of Health, a). COVID-related mandates that Maryland LTC settings were required to meet were 


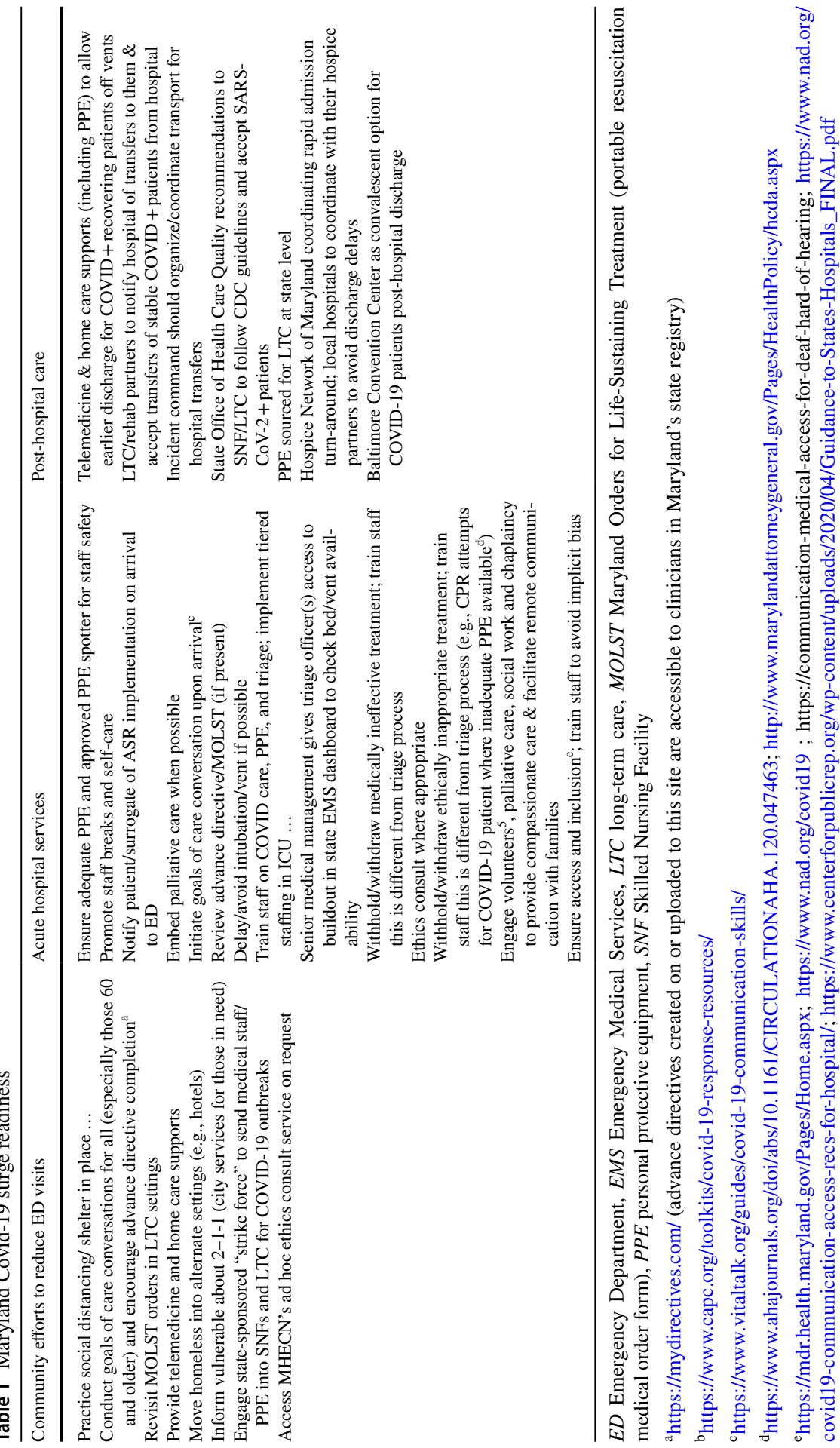


formidable. For example, Maryland's Office of Health Care Quality fined 24 nursing homes for failing to submit daily COVID-19 reporting information required by the Governor's executive order (Tan and Chason, 2020). While improving compliance can be beneficial, there can be unintended consequences. A culture of rigid compliance without exceptions puts additional stress on LTC staff, and this heightened pressure at a time when staff were already over-burdened due to no visitor policies and related COVID-19 infectious control processes may have presented excessive demands. Thus, ASR plans that focus on hospitals-while important—should not detract from how the LTC community can best be supported.

Another example of hospital-centric thinking is that supplies of remdesivir were allocated to hospitals. As a result, individuals with COVID-19 who met remdesivir eligibility criteria and resided in non-hospital settings capable of administering intravenous medication were only able to access the drug to treat COVID-19 if they were transferred to a hospital-indeed, the emergency use authorization from the Food and Drug Administration only allocated it to hospitals [despite research showing it to benefit patients not on mechanical ventilation, earlier in the disease trajectory (Beigel, et al. 2020)]. Allocation strategies that do not take into account the intersection of different types of institutions will prove inadequate at best, and potentially wasteful at worst. This again points to the importance of transparent information sharing and communication so that the different sectors of health care delivery can work together to most appropriately and efficiently distribute valuable resources.

\section{Lesson \#8: The Ability to Correct Course is Crucial: Reconsidering No-visitor Policies}

Evaluating the effectiveness of pandemic responses and their effects on the community is critical. The evolution of no-visitor policies adopted almost universally by health care institutions provides a useful example. The impact of no-visitor policies implemented in hospitals and LTC facilities by the end of March, 2020 amplified the negative effects of social distancing and deeply affected the social well-being of all (Smith et al. 2020). Although important to limiting the spread of COVID-19, these policies also placed a significant burden on patients and their families. The various strategies implemented to keep patients connected to their loved ones, such as telephonic check-ins and/or virtual visits on electronic devices, required enormous amounts of time and effort by the staff to facilitate. WG members shared stories of LTC residents who, in the absence of face-to-face visits, suffered from social isolation, depression, decreased appetite and loneliness. The impact of the no-visitor policies was clearly underestimated and an unintended consequence of good intentions.

Visitors provide support not only to their loved ones but also to the staff. WG members shared how staff at various facilities (both acute and chronic care settings) experienced moral distress, moral injury and burnout due to witnessing the effects of no-visitor policies, despite the time staff spent coordinating patient-family televisits. Stressed staff are more prone to make errors, which can harm patients. Furthermore, stress-associated absenteeism increased the workload for other staff and 
arguably cost facilities extra dollars in contract labor. However, more flexible institutional visitation policies would require adequate personal protective equipment (PPE) and testing and regulations to support this flexibility.

Allowing limited family and others to visit in special situations, for example, at the end of life and during childbirth, was allowed at most institutions. This came with its own challenges. For one, loved ones may have felt pressured to accept that the patient was dying in exchange for the opportunity to visit in person. Many facilities limited the number of visitors who could visit a dying patient, which presented a dilemma for some patients. For example, the presence of a religious practitioner is very important to many, particularly at the end of life (Roman 2020). One WG member reported that his institution designated a faith practitioner as one of the three persons allowed in a dying patient's room at any given time. As Karlawish and colleagues (2020) concluded: “... good policy demands more nuanced thinking about how some visitors contribute" not only to the patient's safety, but also to the holistic care of the individual. Allowing this humane accommodation admittedly requires implementing clear policies and procedures and ensuring access to properly donned PPE. On June 19, 2020, the Maryland Department of Health granted LTC facilities permission to allow LTC residents to visit with loved ones outside of the facility if certain prerequisites were met, including absence of COVID-19 cases among staff and residents, adequate staffing, and adequate availability and use of PPE and SARS-CoV-2 testing capabilities (Maryland Department of Health, b). One can appreciate the burden placed on such facilities to meet these demands.

\section{Conclusion}

The Maryland ASR framework that was published in 2017 recommended that a central state-wide committee be formed to oversee the ASR process in the event of a catastrophic health emergency like the COVID-19 pandemic. Had such a committee been developed, it could have facilitated agreement and coordination across the state to disseminate, modify, and prepare to operationalize the 2017 Plan for use across hospitals. Not only is an ASR plan essential for each hospital, so is a state plan essential to unify and bring order in managing a crisis. This foresight pays multiple dividends. Instead of mounting anxiety and confusion, the public sees public and private sector leaders following predetermined procedures to yield the most favorable outcomes. A state-endorsed ASR plan also minimizes the criticism and opposition hospital executives, department leaders, and incident commanders may face in response to their ASR plan which, in itself, can thwart smooth implementation of the plan. Being prepared allows frontline staff to focus on response and therefore save the most lives.

Even with the Governor's leadership and the results produced by public health and private sector partnerships' universal mitigation and response plans, Maryland has not suppressed the pandemic yet. Over the summer months, public complacency and reduced vigilance allowed the virus to resurge and increased the state's positivity rate over the $5 \%$ rate set by the World Health organization. Fortunately, hospitalizations and the need for ICU services have decreased, but deaths continue to rise. 
With the hospitalizations and ICU admissions of patients with COVID-19 relatively under control (despite recent case increases after the Thanksgiving and Christmas holidays), it is an ideal time to work through the oversight responsibilities, procedural challenges, public messaging, and interdependency needs of a state-endorsed ASR framework. Perhaps this "reality check" could help underscore the importance of infection control and social distancing measures to halt the virus's spread. And even if Marylanders do not perceive immediate benefit to such planning, it is likely that coordinating a central response for pandemic planning that includes a statewide ASR process will help streamline the response to future catastrophic health emergencies in the state.

Acknowledgements We would like to acknowledge the input of other MHECN COVID Working Group members' input into the work that inspired this manuscript.

Authors' Contributions Each author provided substantial contributions to the conception or design of this paper and its drafting and final approval, consistent with the criteria established by the International Committee of Medical Journal Editors (http://www.icmje.org/recommendations/browse/roles-and-respo nsibilities/defining-the-role-of-authors-and-contributors.html).

Funding Not Applicable.

\section{Compliance with ethical standards}

Conflict of interest All authors declare that they have no conflict of interest.

\section{References}

Antommaria, A. H., Gibb, T. S., Mcguire, A. L., \& Wolpe, P. (2020). Ventilator triage policies during the COVID-19 pandemic at US hospitals associated with members of the Association of Bioethics Program Directors. Annals of Internal Medicine, 173(3), 188-194.

Beigel, J.H., Tomashek, K.M., Dodd, L.E., Mehta, A.K., Zingman, B.S. Kalil, A.C., et al. (2020). Remdesivir for the treatment of COVID-19: A preliminary report. New England Journal of Medicine. Available at https://www.nejm.org/doi/pdf/https://doi.org/10.1056/NEJMoa2007764 (Accessed August 29, 2020).

Broadwater, L. (2020). Convention center to become makeshift hospital. The Baltimore Sun, March 25.

Broadwater, L., \& Wood, P. (2020). State prepares for surge. The Baltimore Sun, April 23.

Biddison-Daugherty, L., Gwon, H., Regenberg, A., Schoch-Spana, M., Toner, E. (August 24, 2017). Maryland framework for the allocation of scarce life-sustaining medical resources in a catastrophic public health emergency. Available at: https:/www.law.umaryland.edu/media/SOL/pdfs/Programs/ Health-Law/MHECN/ASR\%20Framework_Final.pdf.

Centers for Medicare \& Medicaid Services. COVID-19 nursing home data (Submitted data as of week ending August 16, 2020). Available at https://data.cms.gov/stories/s/COVID-19-Nursing-HomeData/bkwz-xpvg/ (Accessed August 29, 2020).

Chowkwanuyn, M., \& Adolph, R. (2020). Racial health disparities and COVID-19-Caution and context. New England Journal of Medicine, 383, 201-203.

Daugherty Biddison, E. L., Gwon, H. S., Schoch-Spana, M., Regenberg, A., Juliano, C., Faden, R. R., \& Toner, E. S. (2018). Scarce resource allocation during disasters: A mixed method community engagement study. Chest, 153(1), 187-195.

Daugherty Biddison, E. L., Gwon, H., Schoch-Spana, M., et al. (2014). The community speaks: Understanding ethical values in allocation of scarce lifesaving resources during disasters. Annals of the American Thoracic Society, 11(5), 777-783. 
DHHS (April 8, 2020), OCR reaches early case resolution with Alabama after it removes discriminatory ventilator triaging guidelines. Available at: https://www.hhs.gov/about/news/2020/04/08/ocrreaches-early-case-resolution-alabama-after-it-removes-discriminatory-ventilator-triaging.html (Accessed August 29, 2020).

Ehmann, M.R., Zink, E.K., Levin, A.B., Suarez, J.I., Belcher, H.M.E., Daugherty Biddison, E.L., et al. (in press). How we do it: Operational recommendations for scarce resource allocation in a public health crisis. CHEST. Online version available at https:/www.sciencedirect.com/science/article/abs/ pii/S0012369220348042\#! (Accessed January 3, 2021).

Karlawish, J. Grabowski, D.C., \& Hoffman, A.K. (2020). Continued bans on nursing home visitors are unhealthy and unethical. Washington Post, July 13.

Manchanda, E. C. C., Sanky, C., \& Appel, J. M. (2020). Crisis standards of care in the USA: A systematic review and implications for equity amidst COVID-19. Journal of Racial and Ethnic Health Disparities. https://doi.org/10.1007/s40615-020-00840-5.

Maryland Department of Health (a). Maryland COVID-19 in congregate facility settings: Week of August 26, 2020. Available at https://coronavirus.maryland.gov/pages/hcf-resources (Accessed August 29, 2020).

Maryland Department of Health (b). Amended directive and order regarding nursing home matters. No. MDH 2020-06-19-01. Available at https://phpa.health.maryland.gov/Docum ents/2020.06.19.01\%20-\%20MDH\%20Order\%20-\%20Amended\%20Nursing\%20Home\%20Matters. pdf. (Accessed August 29, 2020)

Mello, M. M., Persad, G., \& White, D. B. (2020). Respecting disability rights -Toward improved crisis standards of care. New England Journal of Medicine, 383(5), e261-e264.

Newey, S. \& Frieden, T. (2020). Blaming CDC for U.S. COVID failures is like blaming someone encased in concrete for failing to swim. The Telegraph, June 28.

Oberlander, J., Marmor, T., \& Jacob, L. (2001). Rationing medical care: Rhetoric and reality in the Oregon Health Plan. CMAJ, 164(11), 1584-1587.

Rodericks, D. (2020). How a west Baltimore nursing home has zero COVID-19 infections. The Baltimore Sun, June 18.

Roman, N. V., Mthembu, T. G., \& Hoosen, M. (2020). Spiritual care - 'A deeper immunity' - A response to Covid-19 pandemic. African Journal of Primary Health Care \& Family Medicine, 12(1), e1-e3. https://doi.org/10.4102/phcfm.v12i1.2456.

Sisak, M. (2020). Field hospitals that went unused to be shut down. The Baltimore Sun, April 30.

Smith, M. L., Steinman, L. E., \& Casey, E. A. (2020). Combatting social isolation among older adults in a time of physical distancing: The COVID-19 social connectivity paradox. Front Public Health, 8, 403.

Sveen, W., \& Antommaria, A. H. M. (2020). Why healthcare workers should not be prioritized in ventilator triage. The American Journal of Bioethics, 20(7), 133-135.

Tan, R. \& Chason, R. (2020). Maryland fines two dozen nursing homes for failing to report covid-19 information, reports deficiencies in others. The Washington Post, June 13.

Tarzian, A. J., Hoffmann, D. E., Volbrecht, R. M., \& Meyers, J. L. (2006). The role of healthcare ethics committee networks in shaping healthcare policy and practices. HEC Forum, 18, 85-94.

Publisher's Note Springer Nature remains neutral with regard to jurisdictional claims in published maps and institutional affiliations.

\section{Authors and Affiliations}

\section{Norton Elson ${ }^{1} \cdot$ Howard Gwon ${ }^{2}$ (D) - Diane E. Hoffmann ${ }^{3}$ (D) Adam M. Kelmenson ${ }^{4}$ (D) . Ahmed Khan ${ }^{5}$. Joanne F. Kraus ${ }^{6}$. Casmir C. Onyegwara ${ }^{7}$. Gail Povar ${ }^{8} \cdot$ Fatima Sheikh $^{9} \cdot$ Anita J. Tarzian ${ }^{10}$}

1 Adventist HealthCare, Gaithersburg, MD, USA

2 Johns Hopkins University Bloomberg School of Public Health, Baltimore, MD, USA 
3 University of Maryland Carey School of Law, Baltimore, MD, USA

4 Faculty of Medicine, Department of Bioethics, The Chinese University of Hong Kong, Shatin, New Territories, HKSAR, China

5 University of Maryland Medical Center, Baltimore, MD, USA

6 Johns Hopkins University School of Medicine (Retired), Baltimore, MD, USA

7 Holy Cross Hospital, Silver Spring, MD, USA

8 GWU School of Medicine and Health Sciences, Washington, DC, USA

9 Johns Hopkins University, School of Medicine, Baltimore, MD, USA

10 Maryland Healthcare Ethics Committee Network, University of Maryland Carey School of Law, Baltimore, MD, USA 